\title{
Mechanism of aortic medial matrix remodeling is distinct in patients with bicuspid aortic valve
}

\author{
Julie A. Phillippi, PhD, ${ }^{\text {a,b,c,d,e }}$ Benjamin R. Green, MS, ${ }^{a}$ Michael A. Eskay, BS, ${ }^{a}$ Mary P. Kotlarczyk, PhD, ${ }^{a}$ \\ Michael R. Hill, PhD, ${ }^{f}$ Anne M. Robertson, PhD, ${ }^{\mathrm{g}}$ Simon C. Watkins, PhD, ${ }^{\mathrm{h}}$ David A. Vorp, PhD, ${ }^{\mathrm{a}, \mathrm{b}, \mathrm{d}, \mathrm{e}}$ and \\ Thomas G. Gleason, $\mathrm{MD}^{\mathrm{a}, \mathrm{b}, \mathrm{c}, \mathrm{d}, \mathrm{e}}$
}

\begin{abstract}
Objectives: Patients with bicuspid aortic valves (BAV) are predisposed to developing ascending thoracic aortic aneurysms (TAA) at an earlier age than patients who develop degenerative TAAs and have a tricuspid aortic valve (TAV). The hypothesis tested is that BAV-associated aortopathy is mediated by a mechanism of matrix remodeling that is distinct from that seen in TAAs of patients with tricuspid aortic valves.
\end{abstract}

\begin{abstract}
Methods: Aortic specimens were collected during ascending aortic replacement, aortic valve replacement, and heart transplants from nonaneurysmal (NA) donors and recipients. Matrix architecture of the aortic media was assessed qualitatively using multiphoton microscopy followed by quantification of collagen and elastin fiber orientation. $\alpha$-Elastin was determined and matrix maturity was assessed by quantifying immature and mature collagen and lysyl oxidase (Lox) expression and activity in aortic specimens. Matrix metalloproteinase-2/9 activity was quantified in aortic smooth muscle cells.
\end{abstract}

Results: Elastin and collagen fibers were more highly aligned in BAV-NA and BAV-TAA cases than in TAV-TAA cases, whereas TAV-TAA cases were more disorganized than TAV-NA cases. $\alpha$-Elastin content was unchanged. Immature collagen was reduced in BAV-NA and BAV-TAA cases when compared with TAV-NA and TAV-TAA cases. Mature collagen was elevated in TAV-TAA cases compared with TAV-NA and BAV-TAA cases. There was a trend toward elevated Lox gene expression and activity and matrix metalloproteinase-2/9 activity for TAV-TAA, BAV-NA, and BAV-TAA specimens.

Conclusions: The highly aligned matrix architecture in patients with BAVs indicates that wall remodeling is distinct from TAV-TAA. Altered matrix architecture and reduced collagen maturity suggest that the effector molecules mediating the remodeling of TAAs are different in BAV and TAV cases. ( $\mathrm{J}$ Thorac Cardiovasc Surg 2014;147:1056-64)

Congenital bicuspid aortic valve (BAV) is associated with an aortopathy manifesting as ascending thoracic aortic aneurysm (TAA) or aortic dissection. BAV is the most common congenital heart anomaly and affects $1 \%$ to $2 \%$ of the population. ${ }^{1,2}$ Data from large surgical centers, including our own, report a BAV occurrence rate of approximately $40 \%$ for patients undergoing aortic replacement as the result of TAA. BAV is heritable, ${ }^{1,3}$ but the cause is unknown. The mechanism mediating the aortopathy

From the Department of Cardiothoracic Surgery, ${ }^{a}$ Department of Bioengineering, ${ }^{\mathrm{b}}$ Center for Thoracic Aortic Disease, ${ }^{\mathrm{c}}$ McGowan Institute for Regenerative Medicine, ${ }^{\mathrm{d}}$ and Center for Vascular Remodeling and Regeneration, ${ }^{\mathrm{e}}$ University of Pittsburgh, Pittsburgh, Pa; Institute for Computational Engineering and Sciences, ${ }^{\mathrm{f}}$ University of Texas, Austin, Tex; and Department of Mechanical Engineering and Materials Science ${ }^{\mathrm{g}}$ and Department of Cell Biology and Physiology, ${ }^{\mathrm{h}}$ University of Pittsburgh, Pittsburgh, Pa.

Funding: The National Heart, Lung, and Blood Institute of the National Institutes of Health, Award Number HL109132 to Dr Gleason and the University of Pittsburgh Medical Center.

Disclosures: Authors have nothing to disclose with regard to commercial support.

Received for publication Feb 26, 2013; revisions received April 12, 2013; accepted for publication April 19, 2013; available ahead of print June 13, 2013.

Address for reprints: Thomas G. Gleason, MD, C-700 Presbyterian University Hospital, 200 Lothrop St, Pittsburgh, PA 15213 (E-mail: gleasontg@upmc.edu). 0022-5223/\$36.00

Copyright (c) 2014 by The American Association for Thoracic Surgery http://dx.doi.org/10.1016/j.jtcvs.2013.04.028 associated with BAV also is not defined, but the pathology is localized distinctly to the ascending thoracic aorta.

A final common pathway of matrix degeneration is shared between aneurysms of the ascending thoracic and abdominal aorta. Increased expression of the degrading matrix metalloproteinases (MMPs) and reduced expression of their counterparts, the tissue inhibitors of matrix metalloproteinases (TIMPs), result in fragmentation of collagen and elastin fibers. Histopathologic observations of cystic medial degeneration (CMD), smooth muscle cell (SMC) loss, lack of inflammatory cells, and proteoglycan accumulation are unique to TAAs and suggest that the inciting mechanism of MMP/TIMP imbalance and matrix degeneration differs for ascending thoracic and abdominal aortic aneurysms.

Patients with BAV are at an increased risk of developing TAA at least 10 to 15 years earlier than patients with TAV. Although the mechanism of action mediating matrix degeneration in BAV-associated aortopathy is unknown, we postulate that SMCs of the BAV aorta possess an inherent defect that causes a material property change in the vessel wall leading to TAA formation. We previously demonstrated that the oxidative stress response is compromised in SMCs in patients with $\mathrm{BAV}^{4}$ We recently reported altered biomechanical strength of the vessel wall between BAV and TAV 

Abbreviations and Acronyms
$\mathrm{BAV}=$ bicuspid aortic valve
CMD $=$ cystic medial degeneration
HYP = hydroxyproline
MMP $=$ matrix metalloproteinase
MPM $=$ multiphoton microscopy
$\mathrm{NA}=$ nonaneurysmal
NOI $=$ normalized orientation index
OI $=$ orientation indice
SMC $=$ smooth muscle cell
TAA $=$ thoracic aortic aneurysm
$\mathrm{TAV}=$ tricuspid aortic valve
TGF $=$ transforming growth factor
TIMP $=$ tissue inhibitors of matrix metalloproteinase

(J Pichamuthu et al, unpublished data). ${ }^{5}$ These data led us to hypothesize that TAAs arise in patients with BAV and TAV by unique mechanisms of matrix remodeling. To test this hypothesis, collagen and elastin matrix architecture and maturity were qualitatively and quantitatively studied in human thoracic aorta from patients with and without aortic aneurysm. We found that patients with BAV and patients with TAV exhibit distinctly different aortic matrix architecture, suggesting distinct mechanisms of aortic wall remodeling in the 2 populations.

\section{MATERIALS AND METHODS}

\section{Patient Selection and Specimen Acquisition}

Ascending thoracic aortic specimens were collected during elective surgery for ascending aortic replacement or aortic valve replacement from patients with BAV or tricuspid aortic valve (TAV) with institutional review board approval and informed patient consent. Specimens were also collected from heart transplant donors and recipients with TAV and absence of aortic disease with approval from the Center for Organ Recovery and Education or institutional review board approval and informed patient consent, respectively. Patient demographics of sex, age, maximal orthogonal aortic diameter, degree of aortic insufficiency and stenosis, and history of hypertension and cigarette smoking were documented (Table 1). Patients with TAA enrolled in our tissue bank with BAV (median age, 53 years; $\mathrm{n}=142$ ) presenting for elective surgery at the University of Pittsburgh Medical Center for ascending aortic replacement do so at least 10 years earlier than patients with TAV (median age 63 years; $n=99$ ). Specimens chosen for each experiment were diameter-matched (median diameters within $2 \mathrm{~mm}$ ) for patients with TAV-TAA and patients with BAV-TAA, and ages span the distribution given above. Specimens of both sexes were included in the study.

On excision, the specimens were placed in ice-cold saline and transported to the laboratory. A portion of tissue was processed for isolation of primary aortic SMC cultures as previously described. ${ }^{4,6}$

\section{Multiphoton Microscopy of Aortic Matrix Architecture}

A $1 \mathrm{~cm}^{2}$ portion of the specimen was dissected, noting proximal/distal orientation. The adventitia was left intact to avoid potential deformation in the native matrix architecture. The specimen was kept in phosphatebuffered saline on ice and imaged immediately (within 2 hours of harvest) using multiphoton microscopy (MPM) or fixed in $2 \%$ paraformaldehyde for 1 hour, followed by storage in phosphate-buffered saline at $4^{\circ} \mathrm{C}$ until MPM was performed. Specimens were imaged using an Olympus FV1000 MPE (Tokyo, Japan) equipped with a Spectra-Physics DeepSee Mai Tai Ti-Sapphire laser (Newport, Mountain View, Calif) using an excitation wavelength of $830 \mathrm{~nm}$ and 1.12 numerical aperture $25 \times$ MPE water immersion objective. Epi-detectors were used to collect the second harmonic generation at $400 \pm 50 \mathrm{~nm}$ for collagen fibers and autofluorescence of elastin at $525 \pm 25 \mathrm{~nm}$. Images were captured using a dwell time of $4 \mu \mathrm{s} /$ pixel and a Kalman filter of 2. Images of the media were captured as $2-\mu \mathrm{m}$ slices starting just beyond the intimal surface sequentially moving toward the adventitia. An attempt to capture images spanning the full thickness of the medial layer was made with each specimen; however, the number of images collected varied with each sample because of thickness and geometry of the specimen and ranged from 14 to $108 \mu \mathrm{m}$.

\section{Determination of Collagen and Elastin Fiber Alignment}

After MPM analysis, projected image stacks were assembled for each channel (collagen and elastin separately) using ImageJ (National Institutes of Health, Bethesda, Md). To quantify collagen and elastin fiber orientation on superimposed 2-dimensional image stacks, the gradient of image intensity at each pixel taken above a threshold was determined for an edge detection algorithm using a custom MATLAB program (The MathWorks Inc, Natick, Mass) previously described. ${ }^{7}$ Briefly, these values were entered into an accumulator bin defined over a subregion, and for each angle the summed gradient-weighted contribution of each pixel was calculated. Histograms of dominant orientation, defined as the angle associated with the maximum accumulator bin value were plotted, and orientation indices (OIs) were determined on the basis of variance of fiber angle $(\Delta \theta)$ of $50 \%$ of the total number of fibers as one half the area under the curve of fiber angle distribution histogram. For images with a high degree of fiber alignment, it was necessary to rotate the image clockwise 90 degrees to generate half-area calculations from fiber-count angle histograms. The normalized orientation index (NOI) was then calculated from Equation 1 as described previously ${ }^{8}$ where $\mathrm{OI}=\Delta \theta$.

$$
\mathrm{NOI}=90-\mathrm{OI} / 90 \times 100 \text { so that } \mathrm{NOI} €[0,100]
$$

(Equation 1)

\section{RNA Isolation and Quantitative Real-Time Polymerase Chain Reaction}

A portion of each specimen was placed in RNAlater (Life Technologies, Carlsbad, Calif) and stored at $-20^{\circ} \mathrm{C}$ until use. Total RNA was isolated using the RNeasy Plus kit (Qiagen, Valencia, Calif). Gene expression of Lysyl oxidase (Lox) was quantified by real-time polymerase chain reaction as previously described ${ }^{6}$ using the One-Step Taqman PCR kit and custom-designed Taqman gene expression assay for human Lox (Assay ID\# Hs00184700_m1) (Life Technologies).

\section{Histology}

Specimens $\left(\sim 0.5 \times 1 \mathrm{~cm}^{2}\right)$ were fixed in $10 \%$ buffered formalin and paraffin embedded. Four-micron sections were stained using Masson's trichrome and Verhoeff-Van Gieson (Research Histology Services, University of Pittsburgh Thomas E. Starzl Transplantation Institute, Pittsburgh, Pa) to ascertain collagen and elastin composition respectively. Slides were stained using picrosirius red stain kit (Polysciences Inc, Warrington, Pa) according to the manufacturer's instructions to assess collagen fiber thickness. Slides were visualized using a Nikon TE-2000-E inverted microscope under polarized light and captured using 
a Nikon DS-Fi1 5MP color camera and NIS Elements Software 3.2 (Nikon Corporation, Melville, NY). Mean area fractions of red/orange and green fibers were quantified.

\section{Determination of Soluble and Insoluble Collagen}

Snap-frozen aortic specimens were pulverized under liquid nitrogen and weighed. Pepsin-soluble collagen was extracted using $5 \mathrm{mg} / \mathrm{mL}$ pepsin in $0.5 \mathrm{~mol} / \mathrm{L}$ glacial acetic acid $(20 \mu \mathrm{L} / \mathrm{g}$ tissue $)$ overnight at $4^{\circ} \mathrm{C}$. Samples were centrifuged at $2100 \mathrm{~g}$ for 6 minutes at room temperature. Undenatured soluble "immature" collagen was detected from the supernatant fraction using the Sircol assay according to the manufacturer's instructions and a standard curve of type I collagen (Accurate Chemical and Scientific Corporation, Westbury, NY) and normalized to tissue mass.

Insoluble collagen content was estimated from the pellet fraction after pepsin digestion using a colorimetric hydroxyproline (HYP) assay. ${ }^{9}$ Pelleted samples with a minimal volume $(<10 \mu \mathrm{L})$ of pepsin solution were lyophilized, massed, and flame-sealed in $10-\mathrm{mm}$ Pyrex tubes with $100 \mu \mathrm{L}$ of $6 \mathrm{~N} \mathrm{HCl}$ containing $0.5 \%$ (v/v) phenol. Samples were incubated at $110^{\circ} \mathrm{C}$ for 24 hours and dried under vacuum. Samples were resuspended in $1 \%(\mathrm{v} / \mathrm{v}) \mathrm{HCl}$, incubated at $50^{\circ} \mathrm{C}$, and an equivalent amount of $\mathrm{NaOH}$. Detection of HYP was achieved by addition of $0.056 \mathrm{~mol} / \mathrm{L}$ Chloramine $\mathrm{T}$ for 25 minutes at room temperature. Freshly prepared $1 \mathrm{~mol} / \mathrm{L}$ Ehrlich's reagent was added, followed by incubation at $65^{\circ} \mathrm{C}$ for 20 minutes. HYP content was estimated from a standard curve of freshly prepared HYP (Sigma, St Louis, Mo). Absorbance was measured at $550 \mathrm{~nm}$ in a spectrophotometer (SpectraMax M2, Model \#D02667; Molecular Devices, LLC, Sunnyvale, Calif) in duplicate wells. HYP values were normalized to the starting mass of the insoluble pellet.

\section{Determination of Elastin Content}

Insoluble $\alpha$-elastin content was determined from aortic specimens using the Fastin Elastin assay (Accurate Chemical and Scientific Corporation) according to the manufacturer's instructions. Tissue samples were pulverized under liquid nitrogen and weighed. Samples were incubated in $750 \mu \mathrm{L}$ of $0.25 \mathrm{~mol} / \mathrm{L}$ oxalic acid in a $100^{\circ} \mathrm{C}$ heating block for 1 hour. Samples were brought to room temperature and centrifuged at $9300 \mathrm{~g}$ for 10 minutes. The supernatant was digested a second time. Alpha-elastin content was estimated from pooled supernatants using a standard curve of $\alpha$-elastin and normalized to weight of the tissue.

\section{Determination of Lox and Matrix Metalloproteinase-2/9 Activity}

For quantification of Lox and MMP 2/9 activity, primary SMCs (passage 4-5) were plated $\left(5000\right.$ cells $\left./ \mathrm{cm}^{2}\right)$ in SMC growth medium (Cell Applications, San Diego, Calif). Media were replenished every other day, and conditioned medium was collected after 7 days. Samples were assayed using the Amplite Fluorometric Lysyl Oxidase Assay Kit (AAT Bioquest, Sunnyvale, Calif) or the Innozyme Gelatinase (MMP-2/9) Activity Assay Kit (EMD Millipore, Billerica, Mass) according to the manufacturers' instructions and reported as fluorescence units normalized to total protein of the conditioned media.

\section{Statistical Analyses}

Data reported represent mean \pm standard error of the mean of all experiments performed. A 1-way analysis of variance was performed followed by Fisher's least significant difference post hoc test to determine differences among patient groups using SPSS 19.0 software (SYSTAT Software, Inc, Chicago, Ill). A Student $t$ test was used to determine diameter matching for TAV-TAA and BAV-TAA within experiments.

\section{RESULTS}

MPM of the ascending thoracic aorta revealed matrix architecture that was unique for patients with BAV and TAV (Figure 1, left). Collagen and elastin fiber architecture appeared to be organized randomly in patients with TAV-TAA versus highly aligned for patients with BAV-nonaneurysmal (NA) and BAV-TAA when compared with TAV-NA. These observations are reflected in the fiber angle count histograms as relatively sharp peaks (parallel, high alignment) for TAV-NA, BAV-NA, and BAV-TAA, or shallow peaks (random, low alignment) for TAV-TAA, and are displayed in Figure 1 (right) for collagen and elastin fibers. Quantification of matrix architecture as a measure of NOI (Figure 2, $A-D$ ) revealed less elastin fiber alignment for TAV-TAA than for TAV-NA $(P=.053)$ (Figure 2,E). A similar trend was observed for collagen $(P=.115)$ (Figure 2, $F$ ).

The matrix architecture of the BAV aorta was striking. In contrast to patients with TAV-TAA, patients with BAV-TAA exhibited a highly oriented fiber architecture that was reminiscent of the architecture observed for a normal aorta with respect to distribution of fiber angle alignment (Figure 1). Elastin fiber alignment in BAV-TAA was increased not only in patients with TAV-TAA but also in patients with TAV-NA $(P<.001)$ (Figure $2, E)$. A similar relationship was determined for the elastin NOI of patients with BAV-NA when compared with TAV-NA $(P=.012)$ and TAV-TAA $(P<.001)$. Collagen NOI was increased in BAV-NA $(P=.009)$ and BAV-TAA $(P=.037)$ when compared with TAV-TAA (Figure 2, $F$ ). Predominant valve pathology (aortic stenosis or aortic insufficiency) or BAV cusp fusion morphology (right-left, right-noncoronary, or true bicuspid) did not affect collagen or elastin NOIs for BAV-TAA (data not shown). Collagen NOI was reduced with increasing aortic diameter for patients with BAV-TAA $\left(\mathrm{R}^{2}=0.73\right)$ but not for patients with TAV-TAA $\left(\mathrm{R}^{2}=0.007\right)$. There was no correlation between elastin

TABLE 1. Study participant demographics

\begin{tabular}{llccll} 
All samples & $\mathbf{N}(\mathbf{M} / \mathbf{F})$ & Age, $\mathbf{y}($ median $\pm \mathbf{S D})$ & Aortic diameter, $\mathbf{m m}($ median \pm SD) & HTN $\%$ (\#/N) & History of smoking $\%(\# / \mathbf{N})$ \\
\hline TAV-NA & $23(17 / 6)$ & $54.0 \pm 14.9$ & Normal & $39.1 \%(9 / 23)$ & $39.1 \%(9 / 23)$ \\
TAV-TAA & $26(22 / 4)$ & $58.5 \pm 11.6$ & $52.0 \pm 7.1$ & $65.4 \%(17 / 26)$ & $23.1 \%(6 / 26)$ \\
BAV-NA & $15(11 / 4)$ & $56.0 \pm 14.4$ & $<40$ & $60.0 \%(9 / 15)$ & $26.7 \%(4 / 15)$ \\
BAV-TAA & $39(35 / 4)$ & $54.0 \pm 9.7$ & $50.0 \pm 5.2$ & $41.0 \%(16 / 39)$ & $59.0 \%(23 / 39)$ \\
\hline
\end{tabular}

$S D$, Standard deviation; $H T N$, hypertension; $A I$, aortic insufficiency; $A S$, aortic stenosis; $T A V$, tricuspid aortic valve; $N A$, nonaneurysmal; $T A A$, thoracic aortic aneurysm; $B A V$, bicuspid aortic valve. 
NOI and aortic diameter for patients with TAV-TAA $\left(\mathrm{R}^{2}=0.26\right)$ and BAV-TAA $\left(\mathrm{R}^{2}=0.002\right)$. Our findings of distinct patterns of collagen and elastin matrix architecture in the aortas of those with BAV and TAV led us to question whether differences in matrix protein maturity exist in BAV and TAV aortas.

Histologic analyses of aortic specimens confirmed CMD, elastin fragmentation, and collagen disorganization in TAA specimens irrespective of aortic valve morphology (Figure 3, A). Nonuniform collagen distribution, elastin fragmentation, and CMD were observed for TAV-TAA, BAV-NA, and BAV-TAA.

There was no difference in $\alpha$-elastin content among the patient groups $(P>.2)$ (Figure 3, $B)$. To assess collagen maturity, soluble collagen "newly synthesized, immature" and insoluble "cross-linked, mature" collagen was quantified. Immature collagen was reduced in BAV-NA and BAV-TAA specimens when compared with TAV-NA and TAV-TAA specimens (Figure $3, C)(P<.02)$ and unchanged between TAV-NA and TAV-TAA specimens $(P=.826)$. There was an elevated amount of mature collagen in TAV-TAA relative to TAV-NA and BAV-TAA $(P<.03)$ (Figure $3, D$ ). Mature collagen appeared to be increased in BAV-NA relative to normal $(P=.105)$. Qualitative (Figure $4, A$ ) and quantitative (Figure $4, B$ ) image analysis of picrosirius red stained sections for each color representing thick (red) and thin (green) fibers suggested there was an increased amount of thicker diameter collagen for TAV-TAA when compared with TAV-NA and BAV-TAA, similar to that of insoluble, mature collagen (Figure 3,D).

To estimate cross-linking capability, Lox gene expression was quantified and revealed a trend of elevated mRNA in TAV-TAA, BAV-NA, and BAV-TAA cases $(P=.079)$ (Figure 5, A), but there was an equivalent level of Lox enzyme activity among all groups $(P=.999)$ (Figure 5 , $B)$. MMP-2/9 activity appeared to be elevated in TAV-TAA, BAV-NA, and BAV-TAA SMCs when compared with TAV-NA $(P=.189)$ (Figure $5, C)$. Gelatin zymography for active MMP-2 yielded a similar pattern (data not shown).

\section{DISCUSSION}

The hallmarks of TAAs in BAV and TAV cases are CMD, elastin fragmentation, and SMC loss. However, TAAs of patients with BAV and TAV are histologically indistinguishable from one another and exhibit patient-topatient variability in matrix organization. Consequently, characterization of valve-specific pathology is difficult. There are disparate reports in the literature on collagen content in various aortopathies. Some reported increased collagen content in thoracic aortic dissections ${ }^{10,11}$ and abdominal aortic aneurysms, ${ }^{12,13}$ whereas others reported no change in collagen for Marfan-related TAA and abdominal aortic aneurysm ${ }^{14}$ or BAV-associated TAA. ${ }^{15}$ Spatially dependent reductions in collagen have been described for patients with BAV-TAA. ${ }^{16}$ We previously observed elevated type I collagen mRNA levels in BAV-TAA versus normal patients ${ }^{6}$ and found total collagen content to be unchanged in BAV-TAA when compared with TAV-TAA (J Pichamuthu et al, unpublished data). In the current study, we demonstrate similar elastin content among all patient groups. Thus, there may be a consensus that differences in BAV versus TAV-associated TAA cannot be reconciled by biochemical changes in the amount of collagen and elastin.

To identify differences in aortic matrix micro-architecture between BAV and TAV, we focused our efforts on a qualitative and quantitative interrogation of collagen and elastin architecture. Other reports have examined aortic matrix architecture in human and animal models using electron microscopy, ${ }^{17,18}$ a time-consuming and destructive process with opportunities for introduction of artifact and screening error. MPM has the capability of visualizing collagen and elastin distinctly in freshly isolated native tissue with minimal, nondestructive processing. We used MPM to interrogate aortic matrix architecture in human aortic specimens from patients with NA and aneurysmal BAV and TAV. The differences in collagen and elastin fiber architecture were striking. Patients with TAV-TAA demonstrated a random, multidirectional organization of collagen and elastin. In contrast, we discovered that patients with BAV displayed a highly aligned unidirectional and parallel fiber architecture. Our findings are consistent with others who proposed that defects in collagen microarchitecture underlie the pathology of thoracic aortic aneurysms related to Marfan syndrome. ${ }^{14}$ Of note, the aorta of patients with abdominal aortic aneurysms and Marfan syndrome was also reported to be stiffer than normal, ${ }^{19,20}$ presumably because of a greater proportion of aligned collagen fibrils. ${ }^{14}$

TABLE 1. Continued

\begin{tabular}{|c|c|c|c|c|c|}
\hline \multicolumn{3}{|c|}{ AI \% (\#/N) } & \multicolumn{3}{|c|}{$\mathbf{A S} \%(\# / \mathbf{N})$} \\
\hline $1+$ & 2-3+ & $4+$ & Mild & Moderate & Severe \\
\hline $0 \%(0 / 23)$ & $4.5 \%(1 / 23)$ & $0 \%(0 / 23)$ & $0 \%(0 / 23)$ & $0 \%(0 / 23)$ & $0 \%(0 / 23)$ \\
\hline $26.9 \%(7 / 26)$ & $23.1 \%(6 / 26)$ & $15.4 \%(4 / 26)$ & $7.7 \%(2 / 26)$ & $0 \%(0 / 26)$ & $3.8 \%(1 / 26)$ \\
\hline $13.3 \%(2 / 15)$ & $6.7 \%(1 / 15)$ & $33.3 \%(5 / 15)$ & $0 \%(0 / 15)$ & $6.7 \%(1 / 15)$ & $53.3 \%(8 / 15)$ \\
\hline $15.4 \%(6 / 39)$ & $23.1 \%(9 / 39)$ & $15.4 \%(6 / 39)$ & $5.1 \%(2 / 39)$ & $10.3 \%(5 / 39)$ & $28.2 \%(11 / 39)$ \\
\hline
\end{tabular}



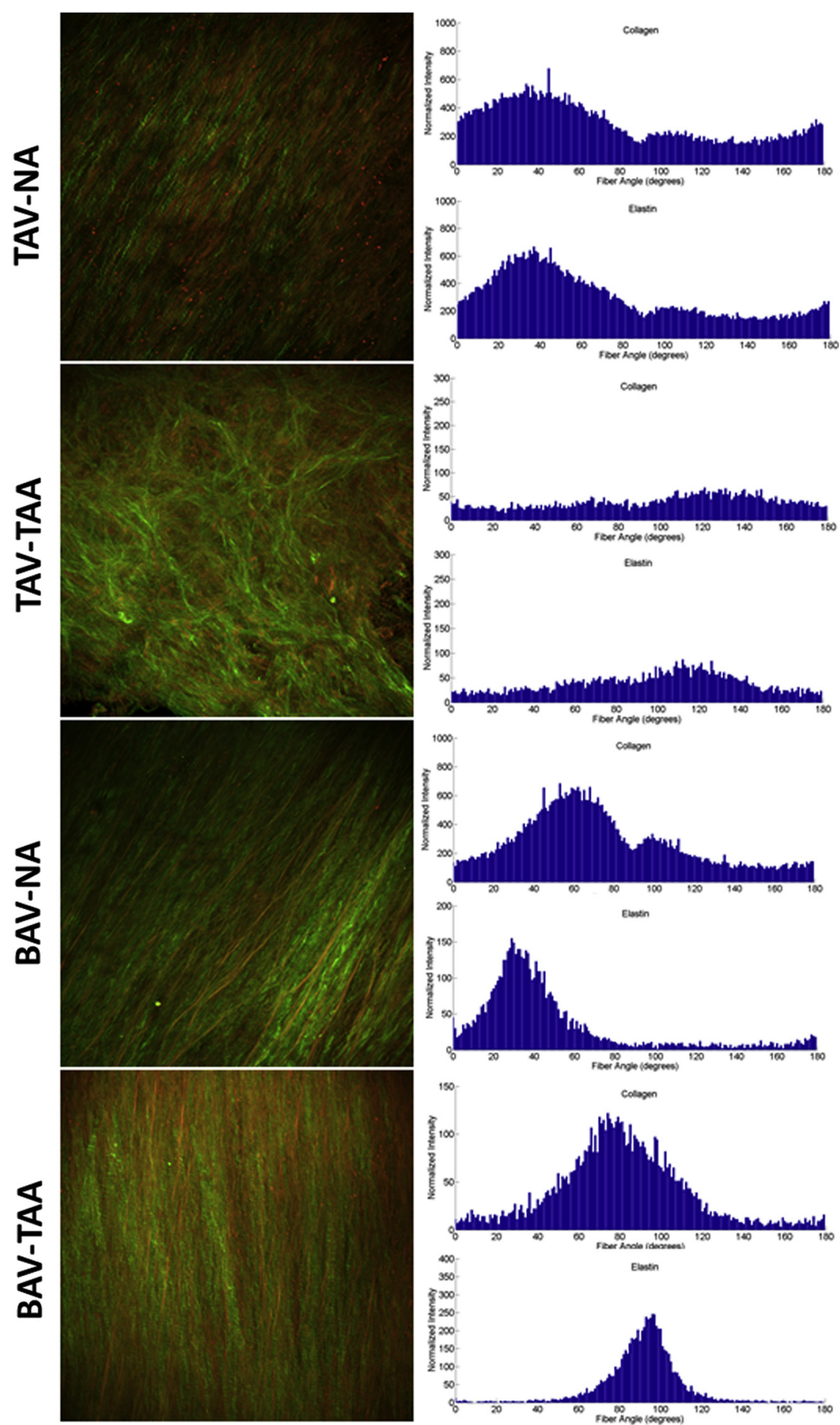

FIGURE 1. Matrix architecture of human ascending thoracic aorta. Representative images of collagen (green) and elastin (red) fiber orientation acquired using MPM (left). Magnification is $25 \times$. Histograms of collagen and elastin fiber angle counts (right). BAV, Bicuspid aortic valve; TAA, thoracic aortic aneurysm; $N A$, nonaneurysmal; $T A V$, tricuspid aortic valve.

The notion of a "microstructural malfunction" rather than a collagen deficit mediating aneurysm formation has been proposed for Marfan-related TAA. ${ }^{14}$ The stark differences in collagen and elastin fiber architecture in $\mathrm{BAV}$ versus TAV indicate that BAV aortopathy involves a remodeling process distinct from that which occurs in 

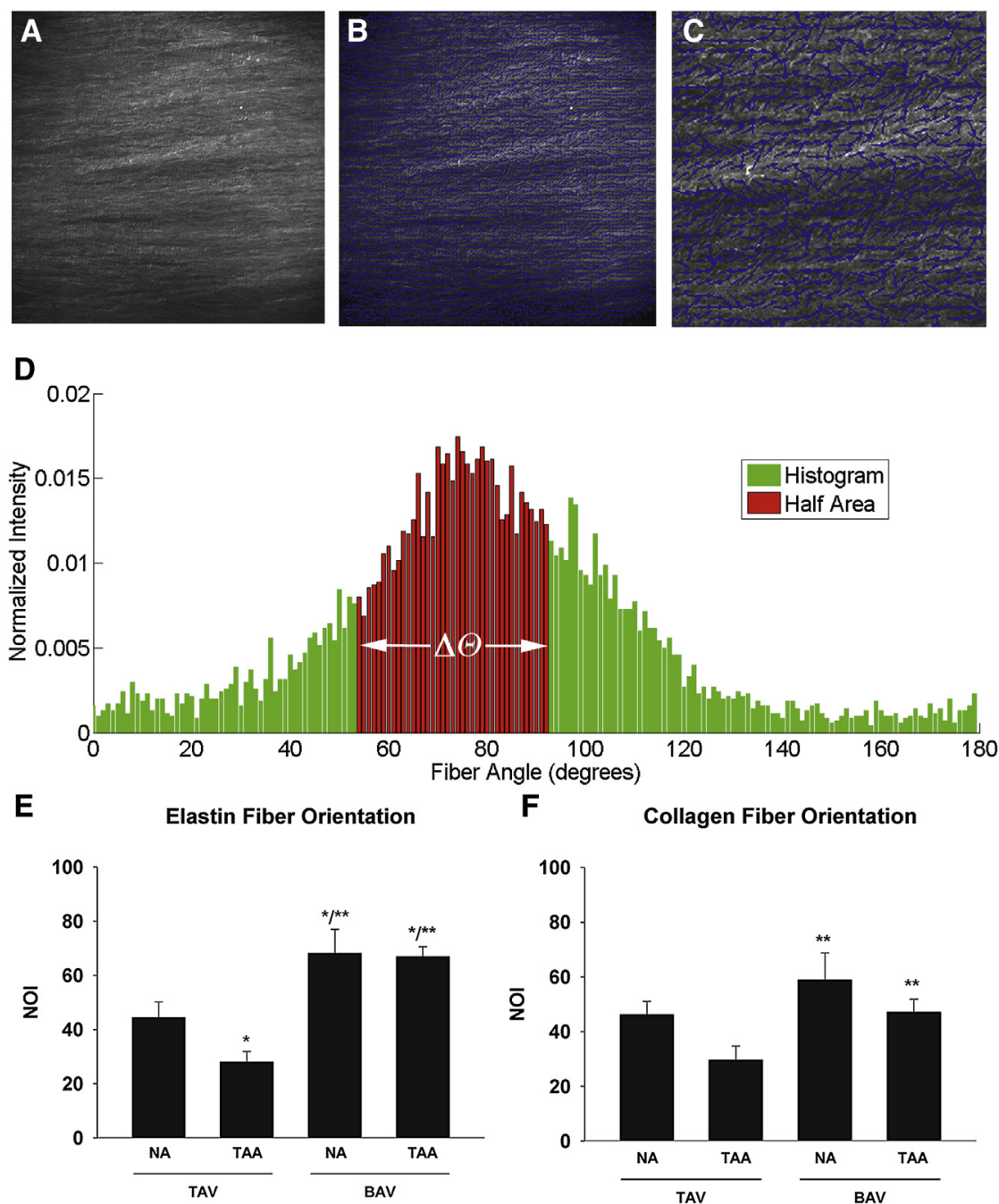

$\mathbf{F}$

Collagen Fiber Orientation

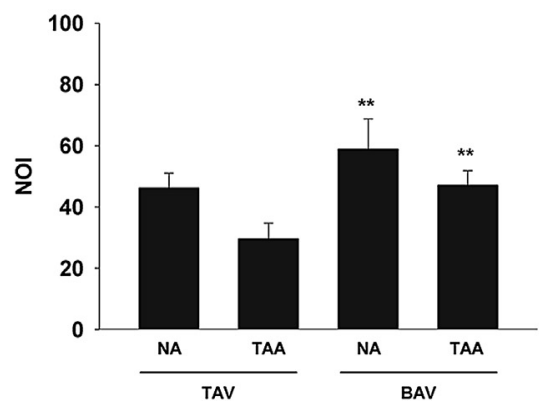

FIGURE 2. Determination of matrix fiber orientation. A, Representative monochromatic image of collagen fiber matrix of a BAV-TAA case using MPM. Magnification is $25 \times$. B, Vector map (blue arrows) of fiber alignment for image in (A). C, Increased magnification of box indicated in (B) to illustrate fiber alignment vectors. D, Representative histogram of collagen fiber angle counts for BAV-TAA generated from MPM image in (A) to calculate the orientation index $(\Delta \theta)$. E and F, NOIs for elastin and collagen, respectively. Bars represent mean \pm standard error of the mean $(S E M), \mathrm{n}=5$ (TAV-NA), 8, 9 (TAV-TAA), 6 (BAV-NA), and 10 (BAV-TAA). *Significant from TAV-NA. **Significant from TAV-TAA, $P<.05$. NOI, Normalized orientation index; $N A$, nonaneurysmal; $T A A$, thoracic aortic aneurysm; $T A V$, tricuspid aortic valve; $B A V$, bicuspid aortic valve.

aneurysms of patients with TAV. Given that patients with BAV-NA also exhibited the same highly aligned fiber orientation as those with BAV-TAA suggests remodeling to be an early event, even before aortic dilatation. These patients with BAV-NA have clinical aortic valve disease and various degrees of aortic stenosis and insufficiency (Table 1). Matrix remodeling in patients with BAV to a more highly aligned fiber architecture appears to be an inherent, early response perhaps as an adaptation to the molecular pathology or turbulent systolic flow jets through the $\mathrm{BAV}^{21}$ The highly oriented collagen fibers may account for our observed differences in vessel strength between TAV-TAA and BAV-TAA ${ }^{5}$ (also J Pichamuthu et al, unpublished data) because aligned collagen fibers tend to be stiffer than randomly oriented fibers, ${ }^{22,23}$ and patients with BAV exhibit increased stiffness of the ascending aorta irrespective of dilatation. ${ }^{24,25}$ Realignment of collagen fibers has been shown to be driven by cell orientation, ${ }^{26}$ supporting our seminal hypothesis that remodeling during TAA in the patient with BAV is cell-mediated. ${ }^{4}$ Furthermore, the recognition of highly aligned elastin fibers is consistent with the known reduced elasticity of BAV-aorta. ${ }^{25}$ The logarithm for determination of collagen and elastin fiber orientation was previously applied to studies of stress-mediated collagen fiber recruitment in rabbit carotid arteries. ${ }^{8}$ Our results follow a similar premise in that collagen fiber alignment in patients with BAV may constitute a remodeling 


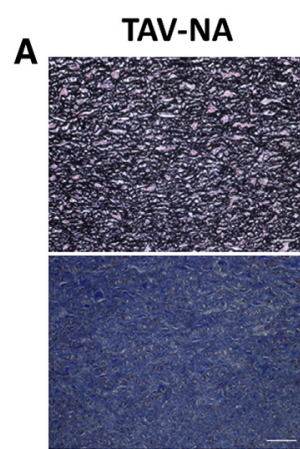

B

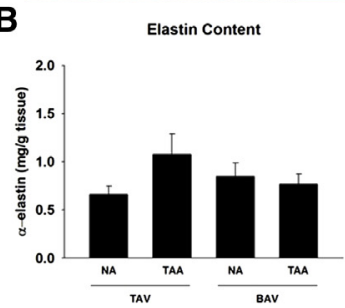

TAV-TAA

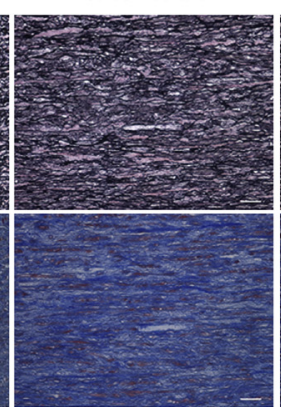

C

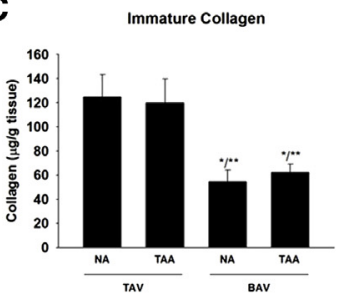

BAV-NA

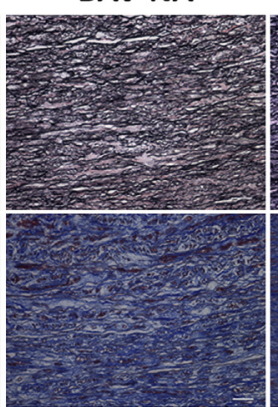

D

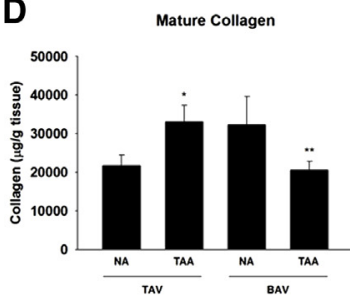

FIGURE 3. Assessment of matrix composition and maturity. A, Verhoeff-Van Gieson (top) and Masson's trichrome (bottom) for representative ascending thoracic aortic specimens. Images were oriented such that the adventitial side is located at the top and intimal side on the bottom. Images were captured at $20 \times$ magnification. Scale bar $=50 \mu \mathrm{m}$. B, Elastin content, $\mathrm{n}=10$ (TAV-NA), 9 (TAV-TAA), 5 (BAV-NA), and 11 (BAV-TAA). C, Immature collagen $\mathrm{n}=8$ (TAV-NA), 9 (TAV-TAA), 4 (BAV-NA), and 9 (BAV-TAA). D, Mature collagen n = 8 (TAV-NA), 7 (TAV-TAA), 3 (BAV-NA), and 8 (BAV-TAA), and bars represent mean \pm SEM. *Significant from TAV-NA. **Significant from TAV-TAA, $P<.05$. TAV, Tricuspid aortic valve; TAA, thoracic aortic aneurysm; $B A V$, bicuspid aortic valve; $N A$, nonaneurysmal.

mechanism in response to circumferential load-bearing that contributes to stress-mediated mechanical stiffening of the vessel. ${ }^{27}$ Collagen fibers were less aligned with increasing aortic diameter for BAV-TAA but not TAV-TAA, suggesting a secondary remodeling mechanism in patients with BAV during progressive dilatation that could be due to local variations in wall stress ${ }^{28}$ and warrants further study. Patients with BAV may have an inability to continually compensate for ongoing degeneration with increasing pressure on the vessel with dilatation. The uniquely remodeled architecture and altered biomechanical responses in those with $\mathrm{BAV}$ and $\mathrm{TAV}^{5}$ (also $\mathrm{J}$ Pichamuthu et al, unpublished data) suggest that different effectors lead to microstructural defects that govern the remodeling process.

Our finding of reduced immature collagen in patients with BAV to TAV irrespective of aortic diameter supports altered collagen remodeling mechanisms at play in the BAV-associated aortopathy. Less immature collagen is consistent with the established pattern of increased MMP activity in BAV-associated TAAs, ${ }^{15,29,30}$ confirmed here in SMCs from patients with TAV-TAA, BAV-NA, and BAV-TAA, because immature, non-cross-linked collagen is more susceptible to proteolysis. An alternative explanation is that reduced immature collagen in BAV could be the result of decreased post-transcriptional modifications or increased pre-pro-collagen protein turnover because we previously observed elevated type I collagen mRNA expression in patients with BAV-TAA. ${ }^{6}$ Mature collagen was increased in patients with TAV-TAA when compared with patients with TAV-NA and BAV-TAA, suggesting a collagen remodeling response in patients with TAV-TAA that is lacking in patients with BAV. Normal levels of immature collagen in TAV-TAA despite elevated MMP activity also points to an altered collagen remodeling response in BAV versus TAV cases. Assessment of collagen fiber diameter via picrosirius red staining concurred with our biochemical analysis of insoluble collagen but did not indicate collagen fiber diameter to be a prominent microstructural alteration for patients with BAV.

These differences in collagen maturity and architecture appear to be independent from cross-linking because Lox gene expression seemed to be elevated similarly in pathologic specimens. The elevated Lox mRNA pattern was congruent to that of type I collagen, further suggesting that the transcriptional machinery responsible for collagen and Lox expression is overstimulated by the same unknown mechanism. Our results complement others who found that Lox and type I collagen were elevated in patients with Marfan syndrome $^{14}$ and postulated the involvement of transforming growth factor (TGF)- $\beta$ in this connective tissue disorder. ${ }^{31}$ TGF- $\beta 1$ is known to regulate collagen and Lox expression and govern post-transcriptional and post-translational processing of Lox. ${ }^{32}$ However, the 
A
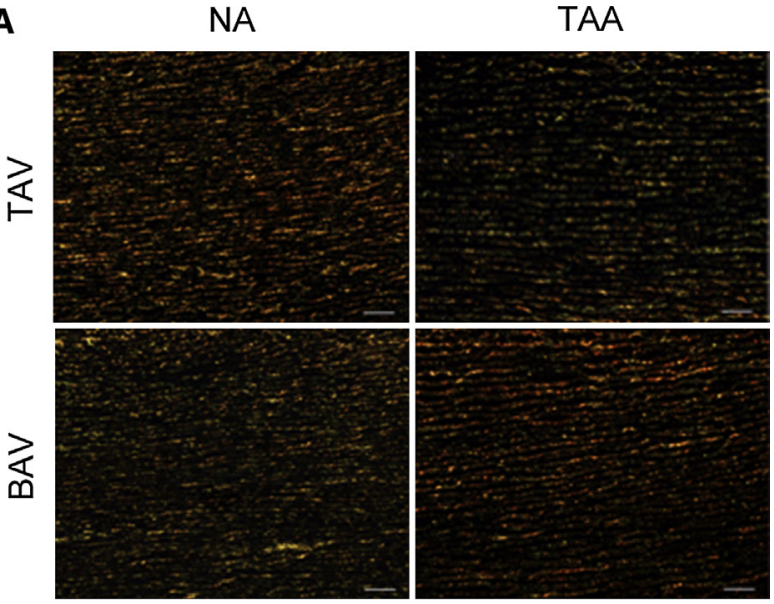

B

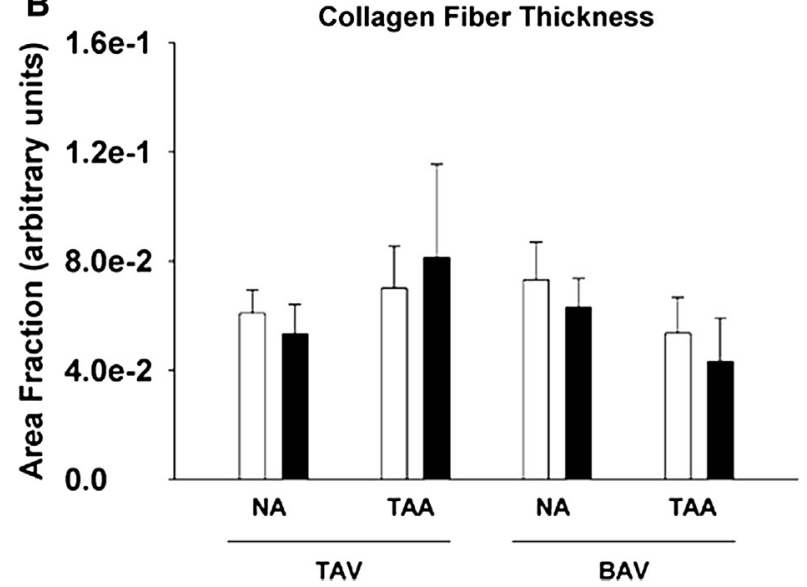

FIGURE 4. Picrosirius red staining for collagen fibers in ascending aorta. A, Representative micrographs of aortic specimens imaged using linear polarized light showing thinner (green) and thicker collagen fibers $(r e d)$. Images were oriented such that the adventitial side is located at the top and the intimal side is located on the bottom. Scale bar $=100$ $\mu \mathrm{m}$. B, Quantification of the area fraction of green (open bars) and red (solid bars). Bars represent mean \pm SEM, $\mathrm{n}=3$ (TAV-NA), 4 (TAV-TAA), and 5 (BAV-NA, BAV-TAA). NA, Nonaneurysmal; $T A A$, thoracic aortic aneurysm; $B A V$, bicuspid aortic valve; $T A V$, tricuspid aortic valve.

role of TGF- $\beta$ in BAV aortopathy needs to be further investigated.

These data collectively lead us to propose that the BAV aorta remodels by a unique mechanism distinct from that giving rise to TAA in patients with TAV. These alterations in matrix architecture in BAV aorta are evident before dilatation and well before the typically recommended point of surgical intervention, which is when the aortic diameter reaches a maximal orthogonal measurement between 50 and $55 \mathrm{~mm}$. It is reasonable to surmise that with an increased understanding of the role that matrix fiber alignment and architecture play in the biomechanical stability of the vessel wall, improved risk assessments for development of TAA and life-threatening dissections can be attained. Further study on the direct impact of the distinct pattern of matrix remodeling in patients with BAV described here on the underlying known differences in biomechanical strength, the putative cell-mediated mechanisms governing the remodeling process, and consideration of the effects of disrupted hemodynamics specific to the pattern of BAV cusp fusion will help glean new insight toward identifying the relative risk for aortic catastrophe in patients with BAV. Interrogation of fetal and pediatric specimens could offer insight on the timing of matrix remodeling and whether the process is adaptive or the distinct differences in architecture are congenital. Ongoing efforts in our laboratory are targeted on characterization of the effector molecules governing the matrix remodeling process in $\mathrm{BAV}$-associated aortopathy.

\section{Study Limitations}

Because of the relatively small portion of tissue that is able to be excised from NA patients with BAV during elective aortic valve replacement, the number of assays that can be performed per patient was limited, which resulted in lower sample sizes for this cohort. The TAV-NA group had appreciably less instances of aortic insufficiency and no instances of AS because the majority of the patients in this cohort were normal "healthy" organ donors, making inferences on the impact of aortic valve pathology on study outcomes difficult.

\section{CONCLUSIONS}

The highly aligned matrix architecture in BAV patients indicates that wall remodeling is distinct from TAV-TAA. Altered matrix architecture and reduced collagen maturity suggests that the effector molecules mediating remodeling of TAA are different in BAV and TAV patients. It is reasonable to surmise that, with an increased understanding of the role matrix fiber alignment and architecture plays in the biomechanical stability of the vessel wall, improved risk assessments for development of TAA and life-threatening dissections can be attained.

The authors thank Erica Butler, Kristin Valchar, and Julie Schreiber for assistance with institutional review board protocols and informed patient consent; Trevor Weis, Deborah Cleary, and Dr John Hempel for assistance with the HYP assay; Jalice Shedrick for assistance with picrosirius red staining; Dr Antonio D'Amore for helpful discussions on calculating fiber orientation; Gregory Gibson for assistance with MPM; Drs Christian Bermudez, Jay Bhama, Forozan Navid, and Lawrence Wei of the Department of Cardiothoracic Surgery, University of Pittsburgh Medical Center, for aortic specimen acquisition; and Dr Phil Campbell for helpful discussions on data analysis and review of statistical analyses. 
A

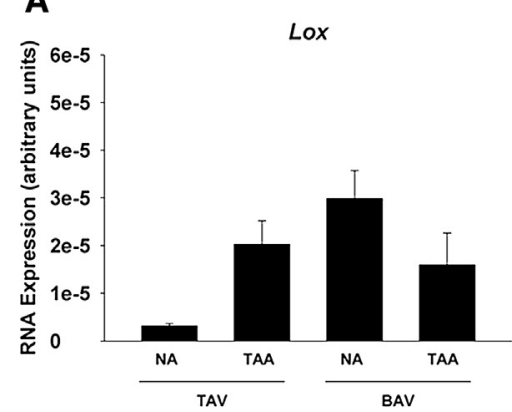

B

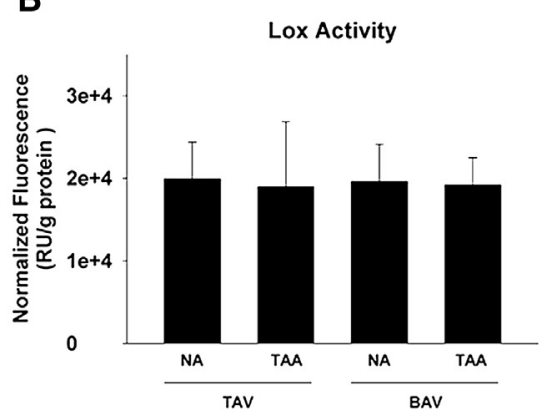

C

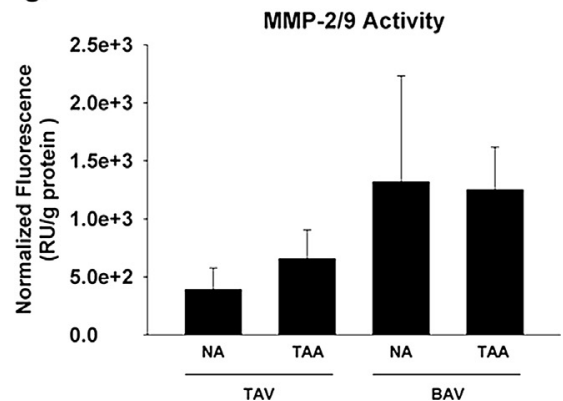

FIGURE 5. Lox and MMP analysis. A, Lox mRNA expression in aortic tissue specimens $n=17$ (TAV-NA), 8 (TAV-TAA), 8 (BAV-NA), and 15 (BAV-TAA). B, Lox $\mathrm{n}=5$ (TAV-NA), 4 (TAV-TAA), 3 (BAV-NA), and 15 (BAV-TAA). C, MMP-2/9 activity n = 4 (TAV-NA), 5 (TAV-TAA), 3 (BAV-NA), and 6 (BAV-TAA). NA, Nonaneurysmal; TAA, thoracic aortic aneurysm; TAV, tricuspid aortic valve; $B A V$, bicuspid aortic valve; $M M P$, matrix metalloproteinase; $N O I$, normalized orientation index.

\section{References}

1. Gleason TG. Heritable disorders predisposing to aortic dissection. Semin Thorac Cardiovasc Surg. 2005;17:274-81.

2. Ward C. Clinical significance of the bicuspid aortic valve. Heart. 2000;83:81-5.

3. Cripe L, Andelfinger G, Martin LJ, Shooner K, Benson DW. Bicuspid aortic valve is heritable. J Am Coll Cardiol. 2004;44:138-43.

4. Phillippi JA, Klyachko EA, Kenny JP 4th, Eskay MA, Gorman RC, Gleason TG Basal and oxidative stress-induced expression of metallothionein is decreased in ascending aortic aneurysms of bicuspid aortic valve patients. Circulation. 2009; 119:2498-506.

5. Pasta S, Phillippi JA, Gleason TG, Vorp DA. Effect of aneurysm on the mechanical dissection properties of the human ascending thoracic aorta. J Thorac Cardiovasc Surg. 2012;143:460-7.

6. Phillippi JA, Eskay MA, Kubala AA, Pitt BR, Gleason TG. Altered oxidative stress responses and increased type I collagen expression in bicuspid aortic valve patients. Ann Thorac Surg. 2010;90:1893-8.

7. Hill MR, Duan X, Gibson GA, Watkins S, Robertson AM. A theoretical and non-destructive experimental approach for direct inclusion of measured collagen orientation and recruitment into mechanical models of the artery wall. J Biomech. 2012;45:762-71.

8. D'Amore A, Stella JA, Wagner WR, Sacks MS. Characterization of the complete fiber network topology of planar fibrous tissues and scaffolds. Biomaterials. 2010;31:5345-54.

9. Stegemann H, Stalder K. Determination of hydroxyproline. Clin Chim Acta. 1967;18:267-73.

10. de Figueiredo Borges L, Jaldin RG, Dias RR, Stolf NA, Michel JB, Gutierrez PS. Collagen is reduced and disrupted in human aneurysms and dissections of ascending aorta. Hum Pathol. 2008;39:437-43.

11. Wang X, LeMaire SA, Chen L, Shen YH, Gan Y, Bartsch H, et al. Increased collagen deposition and elevated expression of connective tissue growth factor in human thoracic aortic dissection. Circulation. 2006;114: I200-5.

12. Menashi S, Campa JS, Greenhalgh RM, Powell JT. Collagen in abdominal aortic aneurysm: typing, content, and degradation. J Vasc Surg. 1987;6: 578-82.

13. Rizzo RJ, McCarthy WJ, Dixit SN, Lilly MP, Shively VP, Flinn WR, et al. Collagen types and matrix protein content in human abdominal aortic aneurysms. J Vasc Surg. 1989;10:365-73.

14. Lindeman JH, Ashcroft BA, Beenakker JW, van Es M, Koekkoek NB, Prins FA, et al. Distinct defects in collagen microarchitecture underlie vessel-wall failure in advanced abdominal aneurysms and aneurysms in Marfan syndrome. Proc Natl Acad Sci U S A. 2010;107:862-5.

15. Fedak PW, de Sa MP, Verma S, Nili N, Kazemian P, Butany J, et al. Vascular matrix remodeling in patients with bicuspid aortic valve malformations: implications for aortic dilatation. J Thorac Cardiovasc Surg. 2003;126: 797-806.

16. Della Corte A, Quarto C, Bancone C, Castaldo C, Di Meglio F, Nurzynska D, et al. Spatiotemporal patterns of smooth muscle cell changes in ascending aortic dilatation with bicuspid and tricuspid aortic valve stenosis: focus on cell-matrix signaling. J Thorac Cardiovasc Surg. 2008;135:8-18, 18 e1-2.

17. Raspanti M, Protasoni M, Manelli A, Guizzardi S, Mantovani V, Sala A. The extracellular matrix of the human aortic wall: ultrastructural observations by FEG-SEM and by tapping-mode AFM. Micron. 2006;37:81-6.

18. Ushiki T. Collagen fibers, reticular fibers and elastic fibers. A comprehensive understanding from a morphological viewpoint. Arch Histol Cytol. 2002;65: 109-26.

19. He CM, Roach MR. The composition and mechanical properties of abdominal aortic aneurysms. J Vasc Surg. 1994;20:6-13.

20. Sonesson B, Hansen F, Lanne T. Abnormal mechanical properties of the aorta in Marfan's syndrome. Eur J Vasc Surg. 1994;8:595-601.

21. Hope MD, Hope TA, Meadows AK, Ordovas KG, Urbania TH, Alley MT, et al. Bicuspid aortic valve: four-dimensional MR evaluation of ascending aortic systolic flow patterns. Radiology. 2010;255:53-61.

22. Brown RA, Prajapati R, McGrouther DA, Yannas IV, Eastwood M. Tensional homeostasis in dermal fibroblasts: mechanical responses to mechanical loading in three-dimensional substrates. J Cell Physiol. 1998;175:323-32.

23. Lo CM, Wang HB, Dembo M, Wang YL. Cell movement is guided by the rigidity of the substrate. Biophys J. 2000;79:144-52.

24. Bilen E, Akcay M, Bayram NA, Kocak U, Kurt M, Tanboga IH, et al. Aortic elastic properties and left ventricular diastolic function in patients with isolated bicuspid aortic valve. J Heart Valve Dis. 2012;21:189-94.

25. Nistri S, Grande-Allen J, Noale M, Basso C, Siviero P, Maggi S, et al. Aortic elasticity and size in bicuspid aortic valve syndrome. Eur Heart J. 2008;29: 472-9.

26. Wang JH, Jia F, Gilbert TW, Woo SL. Cell orientation determines the alignment of cell-produced collagenous matrix. J Biomech. 2003;36:97-102.

27. Okamoto RJ, Xu H, Kouchoukos NT, Moon MR, Sundt TM 3rd. The influence of mechanical properties on wall stress and distensibility of the dilated ascending aorta. J Thorac Cardiovasc Surg. 2003;126:842-50.

28. Nathan DP, Xu C, Plappert T, Desjardins B, Gorman JH 3rd, Bavaria JE, et al. Increased ascending aortic wall stress in patients with bicuspid aortic valves. Ann Thorac Surg. 2011;92:1384-9.

29. Ikonomidis JS, Jones JA, Barbour JR, Stroud RE, Clark LL, Kaplan BS, et al. Expression of matrix metalloproteinases and endogenous inhibitors within ascending aortic aneurysms of patients with bicuspid or tricuspid aortic valves. J Thorac Cardiovasc Surg. 2007;133:1028-36.

30. Boyum J, Fellinger EK, Schmoker JD, Trombley L, McPartland K, Ittleman FP, et al. Matrix metalloproteinase activity in thoracic aortic aneurysms associated with bicuspid and tricuspid aortic valves. J Thorac Cardiovasc Surg. 2004; 127:686-91.

31. Dietz HC, Cutting GR, Pyeritz RE, Maslen CL, Sakai LY, Corson GM, et al. Marfan syndrome caused by a recurrent de novo missense mutation in the fibrillin gene. Nature. 1991;352:337-9.

32. Feres-Filho EJ, Choi YJ, Han X, Takala TE, Trackman PC. Pre- and posttranslational regulation of lysyl oxidase by transforming growth factor-beta 1 in osteoblastic MC3T3-E1 cells. J Biol Chem. 1995;270:30797-803. 